\section{D printed microfluidic devices for cell culture}

Ignazio Roppolo, 1,2

Annalisa Chiappone, 1,2

Candido F. Pirri, ${ }^{1-3}$

Francesca Frascella1,2

1Department of Applied Science and

Technology, Polytechnic University of

Turin, Turin; 2PolitoBIOMed Lab,

Polytechnic University of Turin, Turin;

3Center Advanced Materials for

Sustainable Future Technologies, Italian

Institute of Technology, Turin, Italy

\begin{abstract}
A successful application of the 3D printed materials in the biomedical field requires extensive studies to ensure their biocompatibility at every step of the process. Here, different components suitable for cell applications, including a microfluidic device, were 3D printed using common resins and a deep analysis of their biocompatibility and post printed protocols was conducted.
\end{abstract}

\section{Introduction}

Digital Light Processing (DLP) is widely used in 3D printing, because of its scalability, mild working conditions and fast printing speed. DLP consists in the photopolymerization of photo-curable precursors starting from a CAD project and commonly used inks are acrylates and their derivatives. ${ }^{1}$ Due to its high precision and flexibility, light-based 3D printing techniques represent a valuable instrument for the fabrication of components suitable for biological studies. However, the poor biocompatibility of the inks and the lack of protocols that ensure their sterility slowed down their application in the biological field. Herein, we tested the biocompatibility of three different acrylate-based formulations to assess the feasibility to use them to realize device for cell culture. After adjusting printing parameters, we optimized washing and sterilization protocols to improve cell viability. ${ }^{2}$ In addition, since microfluidic devices enable to control cell culture environment and may be used for drug screening or other cell-based assay to improve reproducibility, ${ }^{3}$ we designed a DLP printed microfluidic device mimicking a 96-well plate, suitable for cell culture and cell-related assays.

\section{Materials and Methods}

Bisphenol A ethoxylate diacrylate (BEDA), 1,6-hexanediol diacrylate (HDDA) and polyethylene glycol diacrylate (PEGDA) with $0,2 \%$ or $1 \%$ of a phosphine oxide-based compound (BAPO) were printed using a PICO 2 DLP-3D printer (Asiga, Australia) with a LED light source (405 $\mathrm{nm}$ ). Washing protocols consisted in incubation or sonication in selected solvents (ethanol or acetone) followed by UV post curing. UV-light, ethanol or autoclave were tested as sterilization methods before cell seeding. Cell viability was assessed using MTT and live and dead assays. ${ }^{3}$ The design of the microfluidic device was done using SolidWorks and the dimension recapitulated the well of a 96-well plate.

\section{Results}

96- and 24-like-wells were 3D printed after the optimization of the printing parameters. The samples were deeply washed, to remove un-reacted materials, evaluating the efficacy of four different post printing protocols. The sonication protocol consisting in sonication for 5 minutes in a solvent (ethanol or acetone) followed by 5 , UV post-curing resulted the most biocompatible. Indeed, incubation seems to be too mild
Correspondence:Marta Canta, Department of Applied Science and Technology, Polytechnic University of Turin, Turin; PolitoBIOMed Lab, Polytechnic University of Turin, Turin, Italy.

E-mail: marta.canta@polito.it

Key words: 3D printing; microfluidics; biomaterials; cytotoxicity; cell culture.

Acknowledgments: The authors acknowledge the funding support by DEFLeCT, Food Drug Free and Smartest project funded by Regione Piemonte POR-FESR 2014-2020.

Disclosures: The authors have nothing to disclose.

Conference presentation: This paper was presented at the Third Centro 3R Annual Meeting - L'era delle 3R: modelli in silico, in vitro e in vivo per promuovere la ricerca traslazionale 30 September - 1 October 2021, Evento online organizzato dal Politecnico di Torino.

Received for publication: 9 July 2021.

Accepted for publication: 7 September 2021.

This work is licensed under a Creative Commons Attribution NonCommercial 4.0 License (CC BY-NC 4.0).

(C) Copyright: the Author(s), 2021

Licensee PAGEPress, Italy

Biomedical Science and Engineering 2021; 4(s1):161 doi:10.4081/bse.2021.161

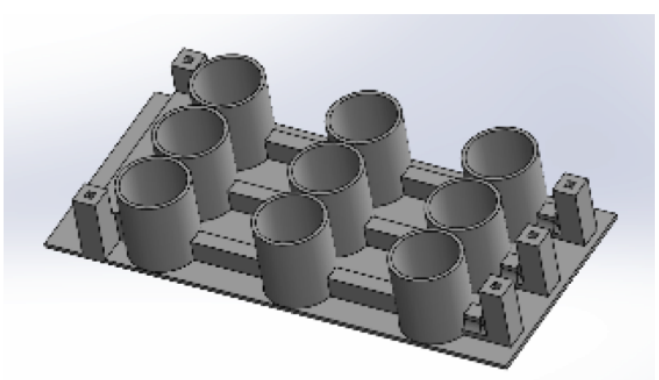

(a)

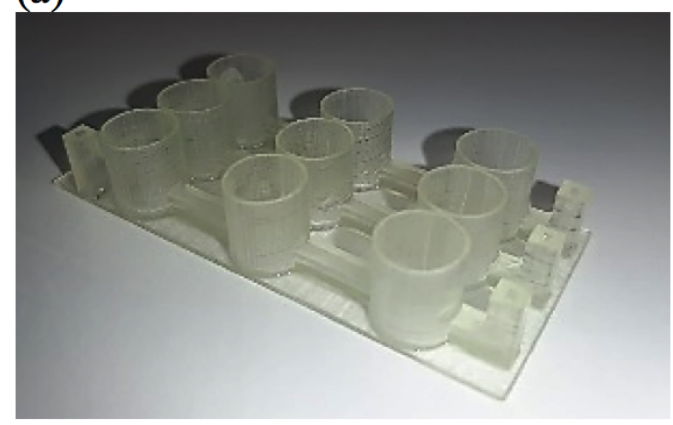

(b)

Figure 1. The CAD design of the microfluidic plate (a) and the 3D printed one (b). 
to remove toxic products leading to a major extent of cell death. Between the different sterilization methods, the ethanol wash allowed to better preserve the mechanical and optical characteristics of the 3D printed wells, without affecting the cell viability. The biological tests identified PEGDA $0,2 \%$ BAPO sonicated in ethanol as the best candidate to be used as main material for a 3D printed device, due to its printability, transparency and cell compatibility [3]. Indeed, BEDA was associated to the lower cell proliferation, while the loss of transparency of the HDDA after the washing step, made it impossible to use for imaging analysis.

Next, the PEGDA formulation was chosen to design a microfluidic plate that recapitulated the 96-well plate dimensions (Figure 1). Fluidic tests showed that the channel succeeded in filling all the wells when connected to a peristaltic pump and the precise design made it suitable to be used in normal plate reader. Finally, viability tests demonstrated that cells can be seeded and grow on the surface of this material assessing its cytocompatibility.

\section{Discussion and Conclusions}

We produced biocompatible 3D printed polymer parts using a commercial DLP-3D printer.

Our results underline the importance of post printing processing to ensure the materials cytocompatibility, needful to proceed with cell cultures tests. Indeed, washing steps are essential in removing non-reacted monomers and photoinitiators to reduce the toxicity of printed parts towards cells. In addition, we demonstrated the feasibility of these materials to produce microfluidic devices for cell cultures.

\section{References}

1. Zhang J, Hu Q, Wang S, et al. Digital light processing based three-dimensional printing for medical applications. Int J Bioprint 2019;6:242.

2. González G, Baruffaldi D, Martinengo $\mathrm{C}$, et al. Materials Testing for the Development of Biocompatible Devices through Vat-Polymerization 3D Printing. Nanomaterials 2020;10:1788.

3. Lee PJ, Ghorashian N, Gaige TA, Hung PJ. Microfluidic System for Automated Cell-based Assays. JALA Charlottesv Va 2007;12:363-7. 